\title{
Zum Verhältnis von kulturellen Werten und gesellschaftlichen Strukturen in der Schweiz - Das Beispiel regionaler Gemeinsamkeiten und Differenzen der Geschlechterungleichheit
}

Ergebnisse eines Forschungsprojektes "Geschlechterungleichheiten in den Regionen

der Schweiz» (Schwerpunktprogramm «Zukunft Schweiz» des Schweizerischen

Nationalfonds)

\section{Elisabeth Bühler, Zürich}

\section{Einleitung}

\subsection{Die Geschlechterdifferenz: Umstrittener Dreh- und Angelpunkt der Gesellschaftsordnung}

Mädchen werden «als Mädchen» und Knaben «als Knaben» erzogen und entwickeln eine persönliche Identität als Frau bzw. als Mann. In vielen gesellschaftlichen Institutionen, z. B. der Familie, der Erwerbsarbeit oder der Politik üben Frauen und Männer weitgehend unterschiedliche Tätigkeiten aus und nehmen unterschiedliche Rollen ein. Bestimmte Verhaltensweisen gelten als männlich, andere dagegen als weiblich und von den Gesellschaftsmitgliedern wird erwartet, dass sie sich an diese (oft ungeschriebenen) Normen halten. Dichotomien wie Verstand - Gefühl, Geist Körper, Öffentlich - Privat, Produktion - Konsum sind prägende Bestandteile unseres (westlichen) Denkens mit einem nicht zuletzt (zwei-)geschlechtlich geprägten Symbolgehalt (McDowell 1999).

In nahezu jeder Kultur werden die dem «männlichen» Pol zugeordneten Bedeutungen zuungunsten des "weiblichen» Pols höher gewertet (HaRDING 1991). Das Geschlecht bzw. die Zweigeschlechtlichkeit ist also ein Dreh- und Angelpunkt der persönlichen Identitätsbildung, der Organisation gesellschaftlicher Institutionen, der Bildung von Herrschaftsformen und der Symbolisierung zentraler Begriffe und Vorgänge.

Die Vorstellung einer bipolaren Geschlechterdifferenz und die Betonung der Verschiedenheit von Frauen und Männern entsprechen jedoch nur einer Möglichkeit des Denkens. Zumindest in der Schweiz und den anderen westlichen Industrieländern hat sich - beginnend mit der Aufklärung im 17. Jahrhundert - im Laufe der Zeit die Erkenntnis durchgesetzt, dass Frauen und Männer im Grunde genommen dieselben Eigenschaften als Menschen besitzen und deshalb auch gleiche Rechte in der Gesellschaft beanspruchen können (Hardmeier 1997, Klinger 1994). Auch zweifeln heute beispielsweise nur noch wenige daran, dass Väter genauso fürsorglich mit ihren Kindern umgehen können wie Mütter oder dass Frauen so gut wie Männer technische Spitzenleistungen vollbringen können. Von führenden Vertreterinnen und Vertretern der Geschlechterforschung wird heute sogar hervorgehoben, dass Frauen und Männer selbst auf der biologischen Ebene mehr Gemeinsamkeiten als Unterschiede aufweisen und die so genannte «natürliche» Differenz im Verhalten und in der Biologie zwischen Frauen und Männern sich bei genauerem Hinsehen oft als kulturelles Konstrukt erweist. Einen guten Überblick über die epistemologische Entwicklung der Frauen- und Geschlechterforschung liefern zum Beispiel WeEdoN (1991) und McDowell (1999).

Das Spannungsfeld zwischen Gleichheit und Differenz in Bezug auf die Bedeutung von Geschlecht prägt die Geschlechterforschung zu Beginn des 21. Jahrhunderts. Einerseits geht es um die Auflösung polarisierter Begriffe und polarer Denkschablonen, anderseits soll die Bedeutung der Kategorie Geschlecht als zentrales gesellschaftliches Sozialisations-, Organisations- und Herrschaftsprinzip (siehe 1. Absatz) sichtbar gemacht werden, womit ungewollt die bipolare Geschlechterdifferenz bestätigt wird. Dieses Paradox gilt es auszuhalten, da es derzeit nicht lösbar ist (BRÜCKNER 1998).

Unumstritten ist dagegen, dass Geschlechterungleichheiten, d.h. direkte oder indirekte Privilegierungen bzw. Benachteiligungen aufgrund der Zugehörigkeit zu einem Geschlecht heute gesellschaftlich nicht mehr legitimierbar sind. Angestrebt werden Chancengleichheit und Gleichstellung der Geschlechter und der Abbau von nach wie vor bestehenden Ungleichheiten (siehe zum Beispiel Schweizerische Bundesverfassung, Artikel 4. Absatz 2).

\subsection{Unterschiede und Gemeinsamkeiten: ein geogra- phischer Blick auf die Geschlechterverhältnisse \\ «How is gender linked to geography? Do men and women live different lives in different parts of the world? And if gendered attributes are socially constructed, then how does femininity and masculinity vary over time and space? What range of variations is there in the social relations between women and men? Are men usually centre-stage and women confined to the margins in all societies?" (McDowel.L. 1999: 1).}

In diesem 1999 veröffentlichten geographischen Lehr- 
buch «Gender, Identity and Place» werden gleich am Anfang verschiedene, zentrale Fragestellungen der geographischen Geschlechterforschung formuliert. Fragen nach den Unterschieden bzw. den Ähnlichkeiten sowohl in der kulturellen Bedeutung von Weiblichkeit und Männlichkeit als auch in den konkreten Geschlechterverhältnissen zwischen verschiedenen Gesellschaften stehen im Zentrum des geographischen Interesses, denn nicht überall kommt dem Merkmal Geschlecht dieselbe identitätsstiftende, strukturelle und symbolische Bedeutung zu.

Geschlecht hat also nicht nur eine Geschichte sondern auch eine Geographie. Die historisch und räumlich vergleichende Perspektive

«... is one of the most efficient ways of challenging relationships that may seem enatural) and taken for granted from the perspective of a particular society and there is scope for a great deal more cross-cultural work.» (McDoWELL 1999: 94).

Gerade angesichts des im ersten Punkt (1.1) geschilderten Spannungsfeldes zwischen Gleichheit und Differenz in Bezug auf die Bedeutung von Geschlecht ist die vergleichende Perspektive mit ihrem Interesse für Ähnlichkeiten und Unterschiede im Geschlechterverhältnis ein gewinnbringender Forschungsansatz (siehe auch DUNCAN 1995). Vergleiche können grundsätzlich für alle räumlichen Massstabsebenen durchgeführt werden, seien es einzelne Stadtquartiere, Regionen, Siedlungstypen, Nationalstaaten oder supranationale Kultur- und Wirtschaftsräume. Welche Massstabsebene(n) einer vergleichenden Untersuchung zugrunde liegen, hängt ausschliesslich von der Fragestellung ab. Der vorliegende Beitrag konzentriert sich auf eine vergleichende Betrachtung der Geschlechterungleichheiten in den Sprachregionen und Siedlungstypen der Schweiz (siehe auch Punkt 2.4).

Mit dieser Hervorhebung der vergleichenden Forschungsperspektive soll jedoch nicht der Eindruck erweckt werden, dass keine anderen Fragestellungen mehr für die geographische Geschlechterforschung von Bedeutung wären. Für einen informativen Überblick über die Vielfalt geographischer Themen aus dem Bereich der Geschlechterforschung sei für den deutschsprachigen Raum auf Aufhauser et al. (1999) sowie Gilbert (1993) verwiesen und für den englischen Sprachraum auf McDowell (1999) und die Women and Geography Study Group (1997).

\section{Theoretische und methodische Hinweise}

\subsection{Grundzüge der Geschlechter-Arrangement Theorie}

Für vergleichende Studien des Geschlechterverhältnisses bildet der von Birgit PfaU-Effinger entwik- kelte Geschlechter-Arrangement Ansatz eine geeignete theoretische Basis (Pfau-Effinger 1996, 1998). Die drei Begriffe Geschlechterkultur, Geschlechterordnung und Geschlechter-Arrangement spielen in diesem Ansatz eine zentrale Rolle.

Der Begriff der Geschlechterkultur bezeichnet die in einer Gesellschaft dominierenden Normen, Werte und Leitbilder zum Verhältnis der Geschlechter in einem bestimmten gesellschaftlichen Bereich (z. B. Familie, Beruf, Sexualität, etc.). Trotz der für moderne Gesellschaften typischen Pluralisierung von Werten und Normen wird in der Geschlechter-Arrangement Theorie davon ausgegangen, dass ungleiche gesellschaftliche Machtverhältnisse zur Folge haben, dass bestimmte kulturelle Leitbilder sich gegen andere im öffentlichen Diskurs durchzusetzen vermögen, und diese dominierenden kulturellen Leitbilder dann auch in den Institutionen verankert werden.

Die Geschlechterordnung umfasst demgegenüber die realen Strukturen des Geschlechterverhältnisses in den verschiedenen gesellschaftlichen Institutionen. Die theoretische Unterscheidung von Geschlechterkultur und Geschlechterordnung ist wichtig, weil sie es ermöglicht, Diskrepanzen im Verhältnis von gesellschaftlichen Leitbildern und gesellschaftlicher Praxis zu erfassen und nach deren Ursachen zu suchen.

Der Begriff des Geschlechter-Arrangements steht für die Art und Weise des Zustandekommens einer bestimmten beobachtbaren Geschlechterordnung. Diese - so die These - ist das Resultat eines Aushandlungs- und Kompromissbildungsprozesses zwischen gesellschaftlichen AkteurInnen. In diesem Aushandlungsprozess werden die geschlechterkulturellen Leitbilder und die Geschlechterordnung miteinander in Beziehung gesetzt und in ein mehr oder weniger hohes Mass an Übereinstimmung gebracht. Diese handlungstheoretische Grundstruktur des Geschlechter-Arrangement Ansatzes erlaubt es, sowohl Frauen wie Männer systematisch als AkteurInnen zu sehen und nicht als passive Opfer struktureller Rahmenbedingungen. Mit Bezug auf Norbert Elias' Begriff der Machtbalance geht Pfau-EFFinger davon aus, dass ein Arrangement im Sinne einer Figuration auch dann stabil sein kann, wenn es auf einer grundlegenden Asymmetrie in den Machtbeziehungen beruht.

\subsection{Die Erfassung der geschlechterkulturellen Werte und Leitbilder}

Die Schweiz kann insofern als «sozialwissenschaftlicher Glücksfall» bezeichnet werden, als dank der grossen Häufigkeit von eidgenössischen Volksabstimmungen umfangreiche Datensätze zu politisch-weltanschaulichen Äusserungen der Bevölkerung existieren, welche sonst in keinem anderen Land der Welt 
zu finden sind (Hermann 1998). Aus den Abstimmungsresultaten kann zwar nicht auf die politisch-weltanschauliche Haltung einer einzelnen Person geschlossen werden, wohl jedoch auf die mittlere Position aller Stimmenden in einem Zählkreis. Durch ein entsprechend vorsichtiges Vorgehen bei der Dateninterpretation lässt sich auch die stets vorhandene Gefahr ökologischer Fehlschlüsse weitgehend ausschliessen (HERMANN 1998).

Über Themen, in denen geschlechterkulturelle Werte und Leitbilder der Bevölkerung explizit zum Ausdruck kamen, wurde in der Schweiz in den letzten Jahrzehnten mehrmals abgestimmt (Aregger 1998, Senti 1994). Diskutiert und einer regional vergleichenden Betrachtung unterzogen werden hier die Abstimmungsresultate von fünf dieser eidgenössischen Urnengänge. Zusätzlich wird mit den regionalen Abstimmungsresultaten ein zusammengesetzter Index der Geschlechterkultur berechnet. Die Berechnung dieses Index erfolgt nach der Methode, welche die UNO (UNDP) für den Human Development Index (HDI) entwickelt hat (UNDP jährlich; siehe auch Perrons 1994). Die Formel bedient sich eines zweistufigen Verfahrens. In einem ersten Schritt wird der Ja-Stimmen Anteil jeder Abstimmung für jede Region nach folgender Formel standardisiert:

$$
\begin{array}{ll}
\mathrm{W}(\mathrm{X}, \mathrm{I}) & =(\mathrm{X}(\mathrm{I})-\operatorname{Min}(\mathrm{X})) /(\operatorname{Max}(\mathrm{X})-\operatorname{Min}(\mathrm{X})) \\
& \text { wobei } \\
\mathrm{W}(\mathrm{X}, \mathrm{I}) \quad \begin{array}{l}
\text { = Standardisierter Wert für die Abstimmung } \\
\text { X für die Region I }
\end{array} \\
\operatorname{Max}(\mathrm{X}) & \begin{array}{l}
\text { höchster Ja-Stimmen Anteil der Abstim- } \\
\text { mung X unter allen Regionen }
\end{array} \\
\operatorname{Min}(\mathrm{X}) & \begin{array}{l}
\text { = niedrigster Ja-Stimmen Anteil der Abstim- } \\
\text { mung X unter allen Regionen }
\end{array} \\
\mathrm{X}(\mathrm{I}) \quad \begin{array}{l}
\text { Ja-Stimmen Anteil der Abstimmung X für } \\
\text { die Region I }
\end{array}
\end{array}
$$

Durch diese Berechnung erhält die Region mit dem höchsten Ja-Stimmen Anteil in der betreffenden Volksabstimmung den standardisierten Wert 1 und die Region mit der tiefsten Zustimmungsrate den standardisierten Wert 0. Die übrigen Regionen erhalten Werte zwischen 0 und 1 . Der zusammengesetzte Index entspricht dem Mittelwert der standardisierten Teilmerkmale (im vorliegenden Fall werden je 5 Teilmerkmale für den Gesamtindex verwendet):

$\mathrm{G}(\mathrm{I})=\left(\left(\mathrm{W}_{1}(\mathrm{X}, \mathrm{I})+\mathrm{W}_{2}(\mathrm{X}, \mathrm{I})+\mathrm{W}_{3}(\mathrm{X}, \mathrm{I})+\mathrm{W}_{4}(\mathrm{X}, \mathrm{I})\right.\right.$ $\left.\left.+\mathrm{W}_{\mathrm{s}}(\mathrm{X}, \mathrm{I})\right) / 5\right)$

wobei

$\mathrm{G}(\mathrm{I}) \quad=$ Gesamtindex für die Region $\mathrm{I}$

Der Gesamtindex kann ebenfalls Werte zwischen 0 und 1 annehmen. Die Region mit dem insgesamt höchsten (=besten) Ja-Stimmen Anteil erhält den höchsten Wert, die Region mit der geringsten Zustimmungsrate den niedrigsten.

\subsection{Die Erfassung der Geschlechterordnung}

In der internationalen Geschlechterforschung besteht weitgehende Übereinstimmung, dass die Institutionen Familien/Haushalte, Erwerbsarbeit/Beruf, Ausbildungssystem sowie Staat/Politik in einer vergleichenden Analyse der Geschlechterordnung auf jeden Fall ins Blickfeld gelangen müssen (z. B. BUNDESAMT Für Statistik (1996), EuropäIsche Kommission (1997), WaLBY (1993)). Diese vier institutionellen Bereiche stehen auch hier im Zentrum der Betrachtung. Sie funktionieren nach je eigenen Regeln und Gesetzmässigkeiten, sind jedoch gleichzeitig auch gegenseitig voneinander abhängig.

In den letzten Jahrzehnten hat in der Geschlechterforschung eine intensive Diskussion über die Aussagekraft statistischer Indikatoren zur Beschreibung von Geschlechterverhältnissen und Geschlechterungleichheiten in den verschiedenen gesellschaftlichen Institutionen stattgefunden (z. B. Perrons (1994), Walby (1994)). Ein wesentliches Resultat dieser Diskussion ist die Erkenntnis, dass die von den statistischen Ämtern zur Verfügung gestellten Daten für aussagekräftige internationale, aber auch intranationale Vergleiche grosse Lücken aufweisen. Die grössten Mängel bestehen im Bereich Familien/Haushalte sowie bei der Erfassung von Arbeiten, die traditionellerweise von Frauen im Privaten und/oder informell geleistet werden. Einige Länder, darunter auch die Schweiz, haben in den letzten Jahren jedoch Anstrengungen zur Verbesserung der Datenlage unternommen (BUNDESAMT FÜr Statistik 1999, Schmid et al. 1999).

Die in diesem Beitrag vorgestellten und diskutierten fünf Merkmale gehören gemäss heutigem Erkenntnisstand und im Rahmen des verfügbaren, regional differenzierbaren Datenangebotes in der Schweiz zum «harten Kern» von Kennzahlen zur Messung und Beurteilung der Geschlechterungleichheit in den Bereichen Familien/Haushalte, Erwerbsarbeit/Beruf, Ausbildungssystem sowie Staat/Politik. Ausgewertet werden Daten der beiden periodisch durchgeführten, grossen eidgenössischen Vollerhebungen (Volkszählung, Betriebszählung) sowie eine vom Soziologischen Institut der Universität Zürich durchgeführte Vollerhebung bei den rund 3000 Schweizer Gemeinden. Analog zum Index der Geschlechterkultur wird mit diesen fünf Merkmalen der Geschlechterordnung auch ein zusammengesetzter Index der Geschlechterordnung berechnet.

\subsection{Die regionalen Gliederungen}

In den nachfolgenden Kapiteln werden die Ergebnisse der statistischen Auswertungen und Berechnun- 
gen für die drei grossen Sprachgebiete

- Deutschschweiz,

- französische Schweiz sowie

- italienische Schweiz

festgehalten. Diese Gebiete werden weiter in die Siedlungstypen

- Stadt (Kernstädte der schweizerischen Agglomerationen),

- Umland (Gemeinden der städtischen Agglomerationsgürtel) sowie

- Land (nicht städtische Gemeinden)

unterteilt. Die Gesamtindices der Geschlechterkultur und Geschlechterordnung werden zusätzlich auch noch für die 26 Schweizer Kantone berechnet und dargestellt.

\section{Ergebnisse}

\subsection{Fünf gleichstellungsrelevante Volksabstimmungen} in der Schweiz

Die fünf gleichstellungsrelevanten Abstimmungsvorlagen, die nachfolgend besprochen werden, betreffen unterschiedliche Aspekte der Geschlechterkultur (Figur 1). Sie sind je in einen spezifischen historischen, gesellschaftspolitischen Kontext eingebettet, dessen wichtigste Merkmale im Folgenden kurz beschrieben werden. (Für ausführliche Darstellungen siehe AREGGER (1998), EIDGENÖSSISCHE Kommission fÜr FrauenFRAGEN (1998), Joris \& WeCKER (1998), SENTI (1994), Voegeli (1997).)

Volksabstimmungen über die Einführung des Frauenstimm- und -wahlrechts in eidgenössischen Angelegenheiten (1959 und 1971). Die Politik war lange Zeit ausschliesslich eine Männerdomäne, nicht nur in der Schweiz sondern in allen modernen Staaten, die im Anschluss an die französische Revolution in Westeuropa entstanden sind. Zwar wurden in jener Zeit unter dem Motto «Freiheit, Gleichheit, Brüderlichkeit» die Privilegien der Feudalherren aufgehoben, als Freie und Gleiche gegenüber dem Staat galten jedoch nur die Männer (Studer 1996). Der explizite Ausschluss aus der Politik bedeutete jedoch nicht, dass das weibliche Geschlecht in den neu gegründeten Staaten des 19. Jahrhunderts funktionslos war. Gemäss den Vorstellungen der Staatsgründer sollten die Frauen in der familiären Privatsphäre das Ihre zum Funktionieren von Staat und Gesellschaft beitragen (SchweIzeriSChe ZeITSChrift Für Geschichte 1996). Legitimiert wurde dieses Gesellschaftsmodell mit der angeblich von Natur aus vorhandenen Eignung «des Mannes» für das Öffentlich-Politische und «der Frau» für das Familiär-Private.

Für die Vertretung und Durchsetzung frauenpolitischer Interessen war der fehlende Status als vollwer- tige Staatsbürgerinnen ein Handicap (STUDER et al. 1998). Zudem ist er grundsätzlich mit den liberalen Idealen der Freiheit und Gleichheit aller Menschen unvereinbar. Diese Benachteiligung wurde deshalb schon früh von Frauen kritisiert und bekämpft (HARDMEIER 1997). In der Schweiz dauerte es jedoch - nach einer gescheiterten Volksabstimmung im Jahre 1959 - bis 1971, bis die Frauen wenigstens bezüglich der politischen Staatsbürgerschaft den Männern rechtlich gleichgestellt waren.

Die Schweiz ist damit das erste Land, das allen Bürgern das Stimm- und Wahlrecht gewährte, gleichzeitig aber auch praktisch des letzte, das dieselben Rechte auch allen Bürgerinnen zugestand (STUDER 1996). Politikwissenschaftlerinnen und -wissenschaftler führen diesen «Sonderfall Schweiz» nicht zuletzt auf Besonderheiten des schweizerischen politischen Systems zurück, insbesondere auf den Föderalismus und die direkte Demokratie.

Volksabstimmung über den Verfassungsartikel «Gleiche Rechte für Frau und Mann» (1981). "Mann und Frau sind gleichberechtigt. Das Gesetz sorgt für ihre Gleichstellung, vor allem in Familie, Ausbildung und Arbeit. Mann und Frau haben Anspruch auf gleichen Lohn für gleichwertige Arbeit.» Am 14. Juni 1981 wurde dieser neue Verfassungsartikel in einer Volksabstimmung von der Schweizer Stimmbevölkerung mit $60 \%$ Ja-Stimmen angenommen. In dieser politischen Auseinandersetzung ging es im Unterschied zur Auseinandersetzung über das Frauenstimmrecht (siehe oben) nicht «nur» um Wertkonflikte über die Rolle der Frauen und Männer in der Gesellschaft, sondern auch um eine materielle Besserstellung der Frauen. Insbesondere die Forderung nach gleichem Lohn für gleichwertige Arbeit bewirkte im Vernehmlassungsverfahren ausgesprochen negative Reaktionen der Arbeitgeberorganisationen (SENTI 1994).

Volksabstimmung über das neue Eherecht (1985). Aus heutiger Sicht ist es nur noch schwer nachvollziehbar, dass in der Schweiz bis vor 15 Jahren (1985) der Ehemann das «Haupt der Familie» und die Ehefrau in mancher Hinsicht den unmündigen Kinder gleichgestellt war (SENTI 1994). Mit einem neuen Eherecht, das Mitte der Achtzigerjahre vom schweizerischen Parlament verabschiedete wurde, sollte dieses alte hierarchische Führungsmodell durch ein neues, partnerschaftliches Leitbild der Ehe ersetzt werden, das dem gewandelten Selbstverständnis der Frauen und den stark veränderten Formen des Zusammenlebens besser entspricht. Unmittelbar nach Verabschiedung der Vorlage im Parlament wurde dagegen jedoch das Referendum ergriffen. Ein rechtsbürgerliches «Komitee gegen ein verfehltes Eherecht» mit Nationalrat Christoph Blocher und dem Schweizerischen 
Gewerbeverein als treibende Kräfte setzte sich zum Ziel, die alte hierarchische Ordnung der Ehe zu verteidigen. Das neue Eherecht wurde als ein gesellschaftspolitisch verfehltes Konzept bezeichnet, das die individuellen Bedürfnisse der Ehegatten über die Bedürfnisse der Familie stelle (SENTI 1994). In der Volksabstimmung vom 22. September 1985 konnte sich das neue Eherecht mit einem Ja-Stimmen-Anteil von $55 \%$ jedoch erfolgreich durchsetzen.

Volksabstimmung über die Einführung einer Mutterschaftsversicherung (1999). Heute gilt in der Schweiz für Mütter ein Arbeitsverbot von 8 Wochen nach der Geburt. Die Fortzahlung des Lohnes für diese Zeit ist jedoch nicht garantiert. Diese hängt davon ab, wie lange eine Frau in einem Betrieb tätig ist, wie viele Tage sie dem Arbeitsplatz wegen Krankheit im laufenden Kalenderjahr bereits fernbleiben musste oder zu welcher Branche das Unternehmen gehört. Nicht selten erhalten Mütter im postnatalen «Mutterschaftsurlaub» deshalb überhaupt keinen Lohn (SCHWEIZERisCher BundesRat 1999). Damit stellt die Schweiz einen (unrühmlichen) Sonderfall in Europa dar.

1999 verabschiedete das eidgenössische Parlament ein Gesetz, das vorsah, erwerbstätigen Müttern einen 14-wöchigen Mutterschaftsurlaub zu gewährleisten mit einem Erwerbsersatz von 80\% des Lohnes. Dagegen wurde in der Folge von einem «Schweizerischen Komitee Nein zur Subventionierung der Mutterschaft» das Referendum ergriffen. Geltend gemacht wurden insbesondere Argumente, welche in dieser «weiteren Aufblähung des Sozialstaates» eine Gefahr für die bestehenden Sozialwerke und eine «Schwächung der Schweizer Wirtschaft» sahen. In der Volksabstimmung am 13. Juni 1999 wurde die Einführung einer Mutterschaftsversicherung mit durchschnittlich $61 \%$ NeinStimmen abgelehnt.

\subsection{Regionale Gemeinsamkeiten und Unterschiede der Abstimmungsresultate}

Am augenfälligsten in Figur 1 sind die Unterschiede der Abstimmungsergebnisse zwischen der deutschen und der lateinischen Schweiz, wobei die beiden Extreme von den städtischen Gemeinden in der französischen Schweiz (Kernstädte und städtisches Umland) und den ländlichen Gemeinden in der Deutschschweiz gebildet werden. Während in den städtischen Gebieten der Westschweiz sämtliche fünf Vorlagen angenommen wurden, lehnten die Stimmenden in den ländlichen Gebieten der deutschen Schweiz ebenfalls sämtliche fünf Vorlagen ab. In den ländlichen Gemeinden der französischen und allen Siedlungstypen der italienischen Schweiz wurden vier von fünf Vorlagen angenommen, in den städtischen Gebieten (Kernstädte und städtisches Umland) der Deutschschweiz drei von fünf. Neben diesen sprachregionalen Diskrepanzen tritt das ebenfalls sichtbare Stadt-Land-Gefälle der Zustimmungsraten zu den fünf Vorlagen etwas in den Hintergrund.

Diese Unterschiede in der Zustimmung zu gleichstellungspolitischen Innovationen sind einerseits ein direkter Ausdruck unterschiedlicher geschlechterkultureller Werte und Leitbilder der (Stimm-)Bevölkerung. Sei es in Bezug auf die Gewährleistung gleicher formaler politischer Rechte, das Ziel einer tatsächlichen, materiellen Gleichstellung der Geschlechter in Beruf, Ausbildung und Familie oder die soziale Absicherung des Verdienstausfalls bei Mutterschaft:stets wird im Abstimmungsverhalten der Bevölkerung in den nicht deutschsprachigen Landesteilen im Durchschnitt ein stärkerer Wille zum Abbau bestehender Geschlechterungleichheiten erkennbar. Dieser auffällige «Röschti-» bzw. «Polentagraben» in den geschlechterkulturellen Werten und Leitbildern wurde unlängst auch durch eine inhaltsanalytische vergleichende Untersuchung der Berichterstattung von Schweizer Tageszeitungen über gleichstellungspolitische Abstimmungsvorlagen belegt (AREGGER 1998: 263).

Anderseits können die vorliegenden unterschiedlichen regionalen Abstimmungsergebnisse auch Ausdruck unterschiedlicher regional vorherrschender politischer Haltungen sein, welche sich nicht direkt auf die Geschlechterkultur sondern auf andere Dimensionen der Weltanschauung beziehen. Zur Diskussion stehen hier in erster Linie die «klassischen» weltanschaulich-politischen Dimensionen «links - bürgerlich» und «liberal - konservativ». Wie eine detaillierte Untersuchung zum Abstimmungsverhalten ergeben hat, harmonieren insbesondere linke und links-liberale Positionen am besten mit den gleichstellungspolitischen Vorlagen der letzten Jahrzehnte (HermanN 1998). In derselben Untersuchung wurde festgehalten, dass die (Stimm-)Bevölkerung der lateinischen Schweiz im Durchschnitt öfters eine linke Weltanschauung vertritt als die Deutschschweizer Bevölkerung (ähnlich KRIESI et al. (1996)) und die Kombination «links-liberal» vor allem für die Grossstadtregionen typisch ist.

Die Diskrepanzen in den Ja-Stimmen Anteilen zu gleichstellungspolitischen Vorlagen zwischen der lateinischen und der deutschen Schweiz können jedoch keinesfalls ausschliesslich mit einer regional unterschiedlichen Verbreitung dieser «klassischen» Weltanschauungen begründet werden, denn in der französischen Schweiz sind beispielsweise selbst katholisch-konservative Zeitungen von Anfang an für die Einführung des Frauenstimmrechts eingetreten, während selbst liberale Zeitungen der Deutschschweiz diesem Anliegen anfänglich kritisch gegenüber standen (Aregger 1998). 
Frauenstimmrecht $1959 \square$ Gleichstellungsartikel $1981 \square$ Mutterschaftsversicherung 1999

Frauenstimmrecht $1971 \square$ Neues Eherecht 1985

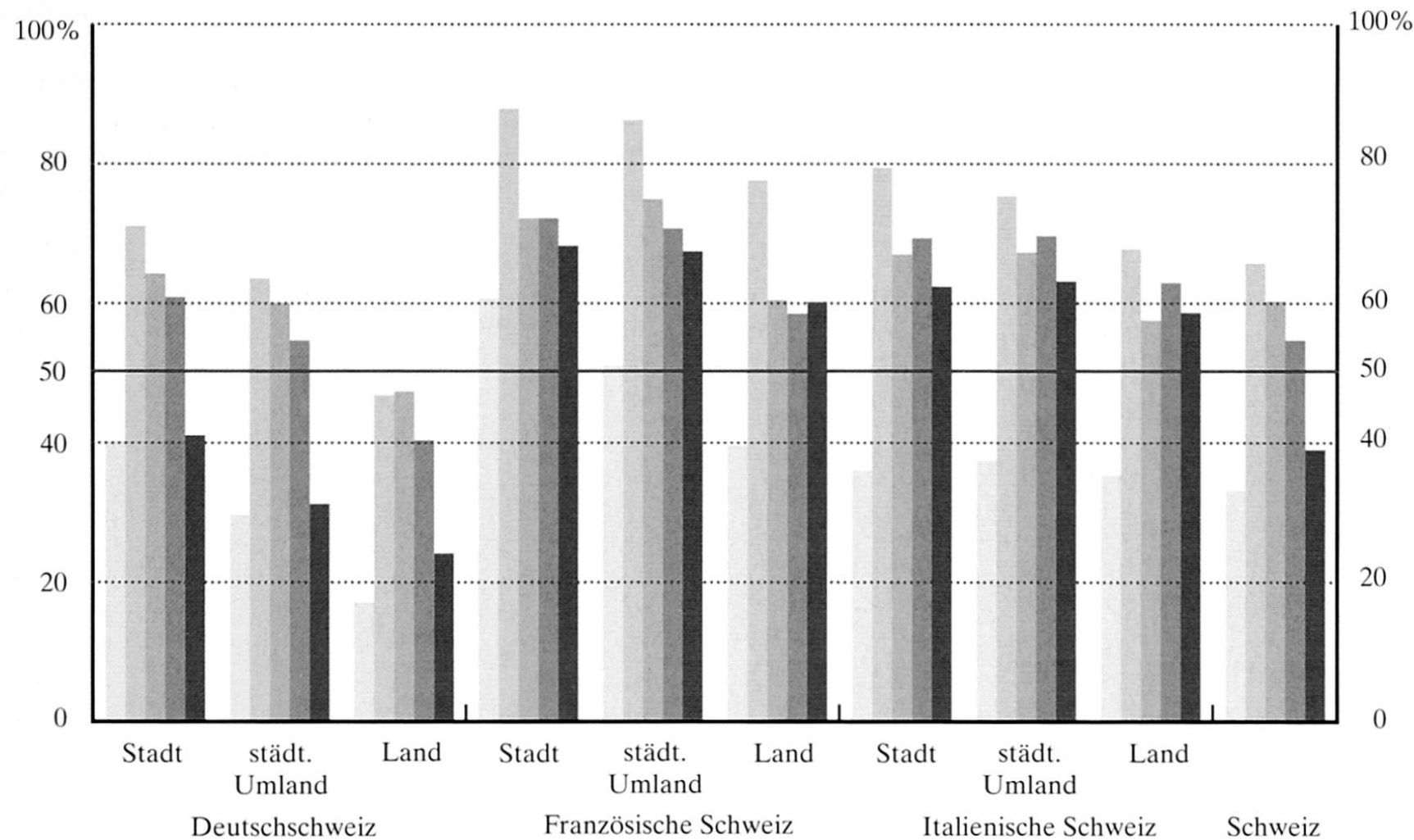

Fig. 1: Ergebnisse gleichstellungsrelevanter Volksabstimmungen; Anteil Ja-Stimmen am Total der gültigen Stimmen nach Sprachregionen und Siedlungstypen in der Schweiz

Results of federal votes on gender relevant issues; proportion of yes-votes by linguistic regions and settlement types in Switzerland

Résultats des votations fédérales sur des thèmes d'égalité entre femmes et hommes; proportion des oui par régions linguistiques et types de commune en Suisse

Daten: Bundesamt Für Statistik, Sektion Politik, Kultur und Lebensbedingungen

Es kann somit festgehalten werden, dass in Bezug auf die dominierenden geschlechterkulturellen Werte und Leitbilder innerhalb der Schweiz deutliche regionale Unterschiede bestehen. Welche sozio-ökonomischen Prozesse und politischen Traditionen diesen Differenzen zu Grunde liegen, geht aus den vorliegenden Daten nicht hervor. Die Beantwortung dieser Fragestellungen muss weiterführenden Untersuchungen vorbehalten bleiben.

\subsection{Fünf zentrale Merkmale der Geschlechter- ungleichheit}

Arbeitsmarkt: Frauenanteil am gesamten bezahlten Beschäftigungsvolumen 1995 und Frauenanteil an den DirektorInnen und leitenden Angestellten 1990. Zur Messung der Geschlechterungleichheiten auf dem Arbeitsmarkt gelangen zwei Kennzahlen zur Anwendung (Figur 2). Der erste Indikator «Frauenanteil am gesamten Beschäftigungsvolumen" gibt Auf- schluss über die quantitative Präsenz der Frauen auf dem Arbeitsmarkt. Ausgedrückt wird das Beschäftigungsvolumen in sogenannten Vollzeitäquivalenten. Eine Person mit einem Teilzeitpensum von $30 \%$ einer vollen Stelle zählt beispielsweise 0.3 Vollzeitäquivalente. Für die Beurteilung der Geschlechterungleichheit im Erwerbsleben ist der Frauenanteil am Beschäftigungsvolumen eine wichtige Kenngrösse. Sie liefert erste Anhaltspunkte, welcher Teil der bezahlten Arbeit den Frauen zufliesst und welcher den Männern. Die quantitative Präsenz der Frauen auf dem Arbeitsmarkt ist jedoch nur ein Merkmal der Geschlechterungleichheit im Erwerbsleben, denn sie sagt beispielsweise noch nichts aus über Einkommen, Arbeitsinhalte, berufliche Entscheidungskompetenzen, Sicherheit des Arbeitsplatzes und Anstellungsbedingungen (regelmässige Arbeit, Arbeit auf Abruf, etc.). Ein aussagekräftiger und für differenzierte regionale Vergleiche zur Verfügung stehender Indikator ist zum 
Beispiel der Frauenanteil an den Personen in höheren beruflichen Stellungen (DirektorInnen und leitende Angestellte).

Familie: Anteil des traditionellen bürgerlichen Familienmodells (Frau nicht erwerbstätig, Mann vollerwerbstätig) am Total der Familien mit Kindern unter sieben Jahren 1990. Der hier gewählte Indikator bezieht sich auf den Anteil der nach dem Modell der traditionellen bürgerlichen Familie (Frau nicht erwerbstätig, Mann Vollzeit erwerbstätig) lebenden Paare am Total aller Paarhaushalte mit Kindern unter sieben Jahren. Unter der Vielzahl heute in der Schweiz gelebter Familienmodelle (BüHLER 2001) repräsentiert das traditionelle bürgerliche Modell die vergleichsweise grössten Geschlechterungleichheiten. Frau und Mann sind in diesem Modell in hohem Masse voneinander abhängig. Dabei ist die Abhängigkeit keine symmetrische und gegenseitige, sondern eine einseitige und hierarchische. Durch die Abhängigkeit vom Einkommen ihres Partners verfügt die Frau über weniger materielle Ressourcen und Entscheidungsspielräume als der Mann (Pfau-EfFinger 1999).

Ausbildung: Mittlere Anzahl Bildungsjahre der 30bis 49-jährigen Frauen in Prozent der mittleren Anzahl Bildungsjahre der 30- bis 49-jährigen Männer 1990. Die erworbene schulische und berufliche Ausbildung gehört zu den wichtigsten Faktoren, welche den Handlungsspielraum einer Person bestimmen (LEVY et al. 1998). Ohne eine gute Ausbildung kann jemand kaum mit einer bedeutenden Stellung im Beruf oder einem hohen Einkommen rechnen. Wer über eine gute Bildung verfügt, kann in der Regel auch leichter mit neuen Herausforderungen in der Familie, im Beruf und in der Gesellschaft umgehen. Bildung vermittelt Kompetenzen und Fähigkeiten und hilft den Menschen, sich in der Welt zu orientieren und am kulturellen Leben teilzunehmen. Die erworbene Ausbildung ist deshalb in unserer Gesellschaft nicht nur eine wirtschaftliche, sondern auch eine soziale und kulturelle Schlüsselgrösse.

Staat: Frauenanteil in den Gemeindeexekutiven 1994. Die Nachwirkungen der strikten Trennung von (öffentlicher) Politik und (privater) Familie und der entsprechenden Verortung der Geschlechter (siehe Punkt 3.1) sind - trotz formalrechtlicher Gleichberechtigung - bis zum heutigen Tag spürbar. Einerseits zeigen sie sich auf einer kulturellen Ebene im männlich (teilweise militärisch) dominierten Symbolgehalt politischer Repräsentationsformen und Praktiken (STUDER et al. 1998). Andererseits sind die Zeit- und Organisationsstrukturen des «politischen Geschäftes» nach wie vor so ausgestaltet, dass sie eine gleichzeitige Vereinbarkeit von Familie und Politik nur mit Schwierigkeiten zulassen. Aus diesen Gründen stellen Frauen in den politischen Behörden, insbesondere in den Exekutiven, auch heute noch eine Minderheit dar (MEULI \& LADNER 2000).

\subsection{Regionale Gemeinsamkeiten und Unterschiede der Geschlechterungleichheit}

Arbeitsmarkt: Der durchschnittliche Frauenanteil am Beschäftigungsvolumen beträgt in der Schweiz 35\% mit einem Maximalwert von $41 \%$ in den Kernstädten der Westschweiz und einem Minimalwert von 32\% im städtischen Umland der Deutschschweiz (Figur 2). Trotz der in den letzten Jahren stark gestiegenen quantitativen Präsenz der Frauen auf dem schweizerischen Arbeitsmarkt (BüHLER 2001) entspricht der Frauenanteil am bezahlten Beschäftigungsvolumen somit in keiner Region dem Frauenanteil an der Bevölkerung. Noch grössere Diskrepanzen bestehen beim Frauenanteil in Führungspositionen, der in keiner hier dargestellten regionalen Einheit die 20\%-Marke überschreitet. Der gemeinhin erwähnte Mindestanteil von rund 30\%, der notwendig wäre, um den Minderheitenstatus einer Gruppe in einer Institution aufzuheben (European Commission 1999), wird damit in Bezug auf die Frauenvertretung in Führungspositionen in keiner Region auch nur annähernd erreicht.

Familie: Zwischen 1970 und 1990 ist in der Schweiz der Anteil der nach dem traditionellen bürgerlichen Familienmodell lebenden Paare von rund $75 \%$ auf gut $60 \%$ zurückgegangen (BüHLER 2001) und aktuelle Stichproben belegen, dass dieser Bedeutungsrückgang sich auch in den Neunzigerjahren fortgesetzt hat (Bundesamt für Statistik 2000). Das traditionelle bürgerliche Familienmodell ist in der französischen Schweiz deutlich weniger verbreitet als in den beiden anderen Sprachregionen (Minimum 48\% Kernstädte Westschweiz, Maximum $71 \%$ ländliche Gemeinden italienische Schweiz) (Figur 2).

Ausbildung: Die mittlere Anzahl Bildungsjahre der Einwohnerinnen und Einwohner der Schweiz, die vom Bundesamt für Statistik mit Hilfe der Angaben der eidgenössischen Volkszählung berechnet werden, sind eine einfache und anschauliche Grösse zur Messung des durchschnittlichen Bildungsstandes der Bevölkerung. Unter einer regionalen Perspektive besteht ein enger Zusammenhang zwischen dem Bildungsstand der Frauen und dem Bildungsstand der Männer. Dort, wo die durchschnittliche Anzahl Bildungsjahre der Männer hoch ist, ist sie es auch bei den Frauen. In allen Raumtypen und Regionen weisen die Frauen aber im Durchschnitt weniger Ausbildungsjahre auf als die Männer (Figur 2); dies obwohl sich der Bildungsrückstand der Frauen in den letzten Jahrzehnten in der Schweiz markant verringert hat (BüHLER 2001). Die regionalen Unterschiede der Geschlechterungleichheiten im mittleren Bildungsniveau sind rela- 
Frauenanteil am Beschäftigungsvolumen 1995

Frauenanteil an den DirektorInnen und leitenden Angestellten 1990

Anteil der nach dem Modell «Mann vollzeitarbeitend, Frau nicht erwerbstätig» lebenden Familien am Total der Familien mit Kindern unter 7 Jahren 1990

Mittlere Anzahl Bildungsjahre der 30-49-jährigen Frauen in Prozent des entsprechenden Wertes der Männer 1990

Durchschnittliche Sitzanteile der Frauen in den Gemeindeexekutiven 1994 (Anzahl ausgewertete Gemeinden: 2057)

$100 \%$

80

60

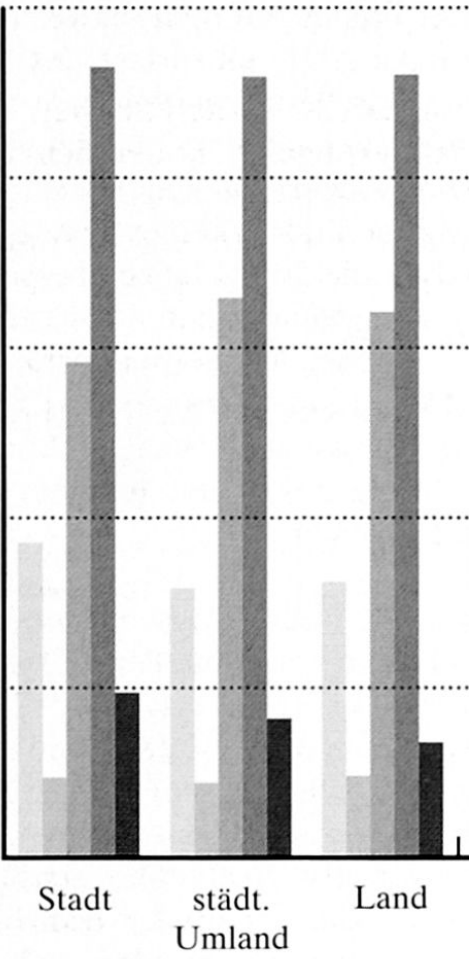

Deutschschweiz

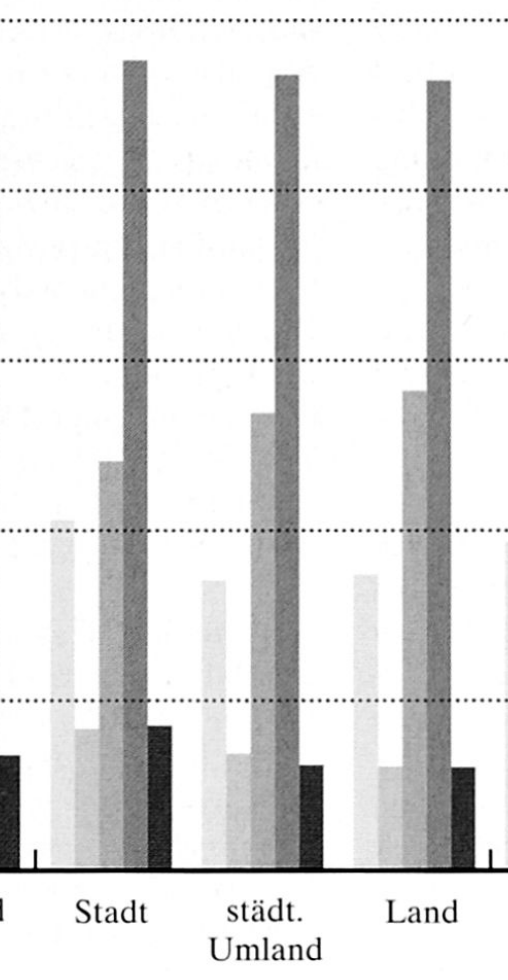

Französische Schweiz

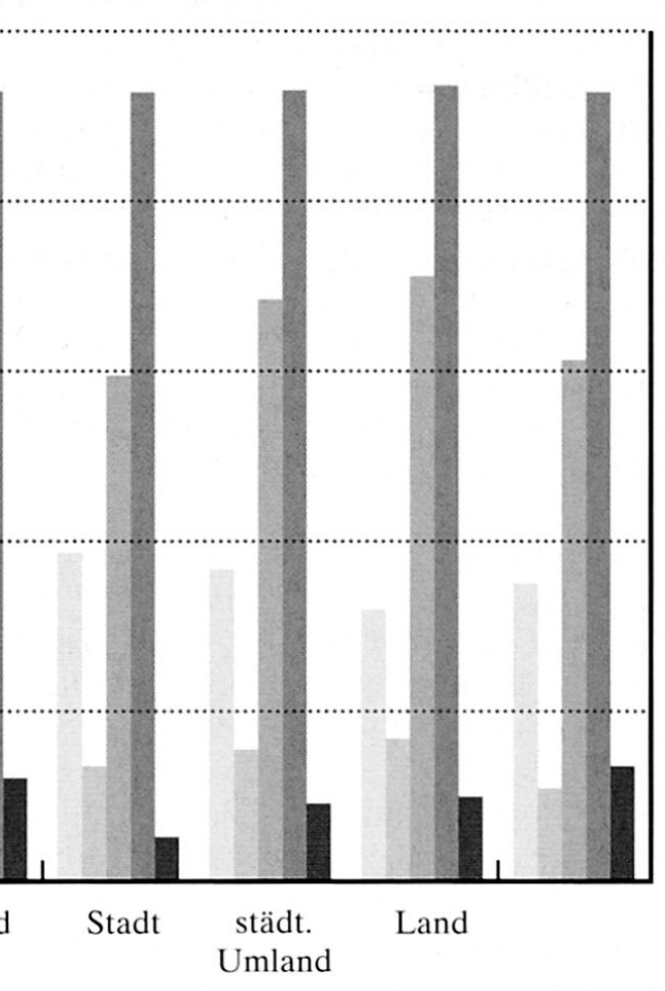

Italienische Schweiz
$100 \%$

Fig. 2: Merkmale der Geschlechterungleichheit nach Sprachregionen und Siedlungstypen in der Schweiz Forms and degrees of gender inequality by linguistic regions and settlement types in Switzerland

Formes et degrés d'inégalité entre les genres par régions linguistiques et types de commune en Suisse Daten: M1: BFS, eidg. Betriebszählung; M2, M3, M4: BFS, eidg. Volkszählung; M5: A. LADNER, Soziologisches Institut, Universität Zürich

tiv klein. Die geringsten Ungleichheiten weisen die Kernstädte der französischsprachigen Schweiz auf, wo die mittlere Anzahl Bildungsjahre der Frauen $95 \%$ des entsprechenden Wertes bei den Männern beträgt. Die grösste geschlechtsspezifische Bildungsdifferenz der Wohnbevölkerung verzeichnet mit einem Werte von $92 \%$ das städtische Umland der Deutschschweiz.

Staat: Im Gegensatz zu den anderen, bereits besprochenen Institutionen kommt der Westschweiz in Bezug auf die Frauenvertretungen in den Gemeinderegierungen keine Vorreiterrolle zu (siehe auch
SEITZ (1994)). Den höchsten mittleren Frauenanteil in den Gemeindeexekutiven verzeichnen diesmal die Kernstädte der Deutschschweiz (19\%), während die Tessiner Kernstädte mit einem durchschnittlichen Frauenanteil von nur 5\% das absolute Schlusslicht bilden (Angaben für die Stadt Lugano fehlen jedoch).

Insgesamt machen die in Figur 2 dargestellten Merkmale der Geschlechterungleichheit deutlich, dass in keiner Sprachregion und keinem Siedlungstyp der Schweiz von einer tatsächlichen Gleichstellung der Geschlechter gesprochen werden kann. Neben dieser 
Gemeinsamkeit treten die durchaus vorhandenen regionalen Unterschiede etwas in den Hintergrund. Die geringsten Geschlechterungleichheiten sind tendenziell in der französischen Schweiz festzustellen. Bei den meisten Merkmalen wird auch ein Stadt-LandGefälle im Gleichstellungsgrad erkennbar.

Gemäss dem Geschlechter-Arrangement Ansatz stellen diese strukturellen Gegebenheiten einen Teil der Geschlechterordnung in der Schweiz dar und sind im Sinne eines Aushandlungs- und Kompromissbildungsprozesses zu interpretieren. In diesem Prozess werden die kulturellen Werte und Normen der beteiligten Akteure und Akteurinnen und die wirtschaftlichen, politischen und familiären Rahmenbedingungen in ein mehr oder weniger hohes Mass an Übereinstimmung gebracht (siehe Punkt 2.1). Der effektive Aushandlungs- und Kompromissbildungsprozess lässt sich mit den vorliegenden sekundärstatistischen Daten jedoch nicht nachvollziehen, weshalb momentan die Frage offen bleiben muss, inwieweit die dargestellten Strukturen der Institutionen Arbeitsmarkt, Familie, Politik und Ausbildungssystem den Werten und Leitbildern der beteiligten Akteure und Akteurinnen entsprechen oder nicht. Um diese komplexen sozialen Prozesse der Konstruktion und Legitimation von Geschlechterungleichheit und die dahinter liegenden Motive und Strategien der handelnden Personen vertiefter zu untersuchen, wären weiterführende, in erster Linie qualitative Untersuchungen geeignet.

\subsection{Fazit: Zum Verhältnis von gesellschaftlichen Strukturen und kulturellen Werten}

Die Gesamtindices der Geschlechterkultur und der Geschlechterordnung sind für die Sprachregionen und Siedlungstypen in Figur 3 und für die Schweizer Kantone in Figur 4 dargestellt. In der Schweiz besteht für beide Regionalisierungen ein deutlich erkennbarer Zusammenhang zwischen regionaler Geschlechterkultur und regionaler Geschlechterordnung (Pearson Produkt-Moment-Korrelationskoeffizient $\mathrm{r}=0.8$ für Sprachregionen/Siedlungstypen und $r=0.7$ für Kantone). Dort, wo die Abstimmungsergebnisse insgesamt eine gleichstellungsfreundlichere Einstellung der

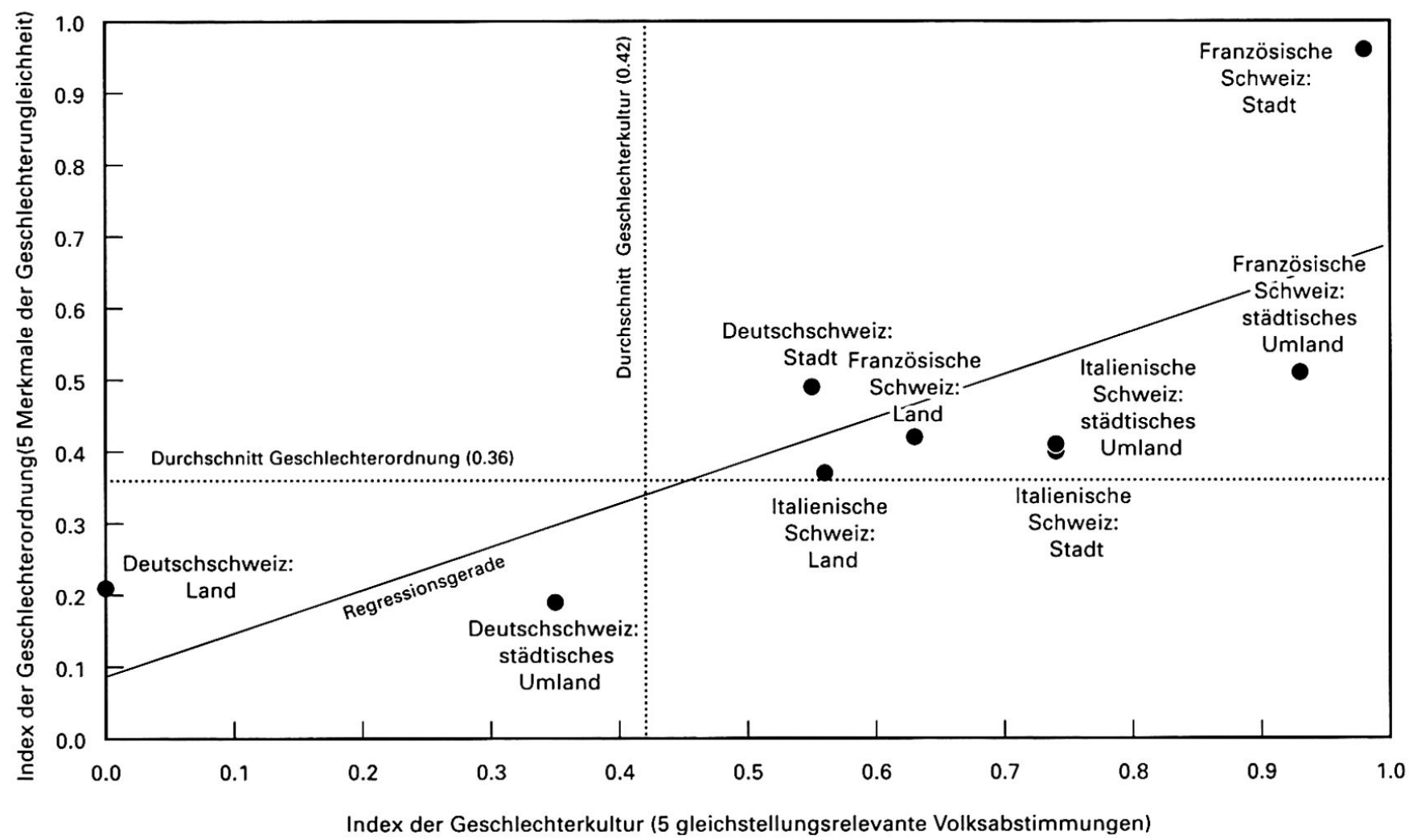

Fig. 3: Index der Geschlechterkultur und Index der Geschlechterordnung nach Sprachregionen und Siedlungstypen in der Schweiz

Index of gender culture and index of gender order by linguistic regions and settlement types in Switzerland Indice de "gender order» et indice de "gender culture» par régions linguistiques et types de commune en Suisse Daten: Bundesamt Für Statistik, Sektion Politik, Kultur und Lebensbedingungen; BFS, eidg. Betriebszählung; BFS, eidg. Volkszählung; A. LADNER, Soziologisches Institut, Universität Zürich 


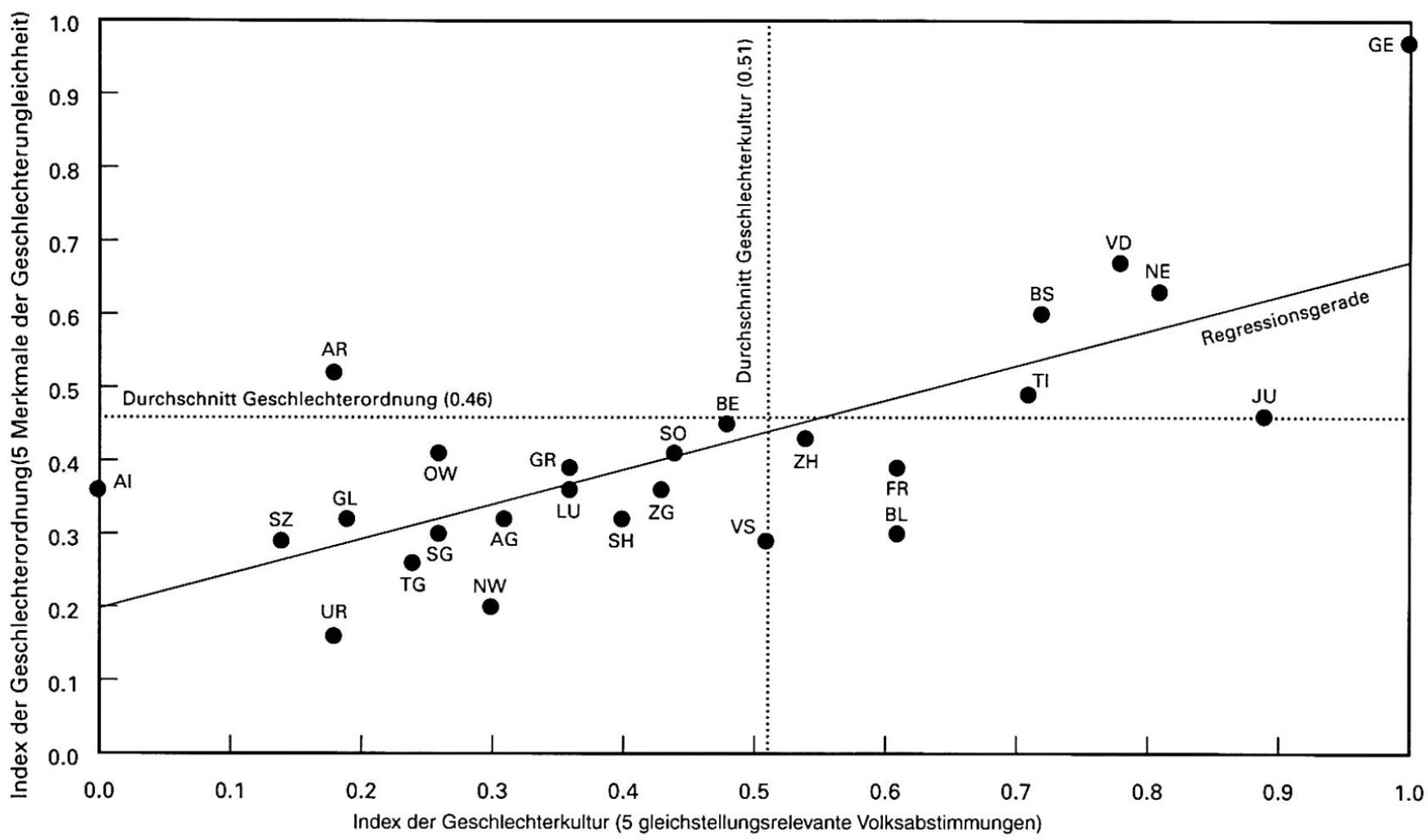

(Legende: AI: Appenzell Innerrhoden, AR: Appenzell Ausserrhoden, SZ: Schwyz, GL: Glarus, UR: Uri, OW: Obwalden, SG: St. Gallen, TG: Thurgau, AG: Aargau, NW: Nidwalden, GR: Graubünden, LU: Luzern, SH: Schaffhausen, SO: Solothurn, ZG: Zug, BE: Bern, VS: Wallis, ZH: Zürich, FR: Fribourg, BL: Basel-Landschaft, TI: Tessin, BS: Basel-Stadt, VD:Waadt, NE: Neuenburg, JU: Jura, GE: Genf)

Fig. 4: Index der Geschlechterkultur und Index der Geschlechterordnung nach Schweizer Kantonen Index of gender culture and index of gender order by Swiss cantons Indice de "gender order» et indice de "gender culture» par cantons suisses Daten: Bundesamt Für Statistik, Sektion Politik, Kultur und Lebensbedingungen; BFS, eidg. Betriebszählung; BFS, eidg. Volkszählung; A. LADNER, Soziologisches Institut, Universität Zürich

Stimmbevölkerung zum Ausdruck bringen, ist auch die Geschlechterungleichheit insgesamt relativ geringer als in Regionen mit einer weniger gleichstellungsfreundlich eingestellten Stimmbevölkerung. Diese Kombination trifft am ausgeprägtesten auf die Kernstädte der französischen Schweiz (Figur 3) bzw. den Kanton Genf (Figur 4) zu. Überdurchschnittlich hohe Werte beider Indices weisen daneben auch die Kantone Waadt (VD), Neuenburg (NE), Basel-Stadt (BS) und Tessin (TI) auf sowie alle in Figur 3 dargestellten Regionstypen mit Ausnahme der ländlichen Gebiete und des städtischen Umlandes in der Deutschschweiz. Deshalb erstaunt es wenig, dass in Figur 4 vor allem viele Kantone der eher ländlich geprägten Deutschschweiz im unteren linken Quadranten liegen.

Trotz dieses insgesamt deutlichen Zusammenhangs zwischen regionaler Geschlechterkultur und regionaler Geschlechterordnung fallen in beiden Figuren regio- nale Kontexte mit Diskrepanzen zwischen Geschlechterkultur und Geschlechterordnung auf. So steht beispielsweise der Kanton Jura (JU) auf Rang zwei, was die Zustimmungsquoten zu gleichstellungspolitischen Vorlagen anbetrifft; hinsichtlich der tatsächlichen Gleichstellung der Geschlechter nimmt dieser Kanton jedoch nur den siebten Rang unter den 26 Kantonen ein. Eine ähnliche Diskrepanz - relativ gleichstellungsfreundliches Abstimmungsverhalten jedoch relativ hohe strukturelle Geschlechterungleichheit - ist für die beiden städtischen Gebietstypen der italienischen Schweiz festzustellen (Figur 3). Aber auch das Umgekehrte kann der Fall sein: Der Kanton Appenzell Ausserrhoden (AR) steht zwar auf dem fünftbesten Rang der strukturellen Gleichstellungsskala, gleichzeitig aber auf dem drittletzten Rang, was die Zustimmungsquoten zu den gleichstellungspolitischen Abstimmungsvorlagen anbetrifft. Worauf diese Diskrepanzen zwischen Geschlechterkultur und Geschlechterordnung 
im Detail zurückzuführen sind, müsste für jeden Einzelfall genauer betrachtet werden.

Nicht alle urbanen Regionen der Schweiz bieten unter dem Aspekt der Gleichstellung für Frauen grundsätzlich bessere Lebensbedingungen an als ländliche (Figur 3). Besonders die städtischen Gebiete der Deutschschweiz schneiden unter diesem Gesichtspunkt ungünstig ab. Sie liegen sowohl hinsichtlich der Geschlechterordnung, insbesondere jedoch hinsichtlich der dominierenden geschlechterkulturellen Werte deutlich hinter den städtischen Gebieten der anderen Sprachregionen zurück. Selbst die ländlichen Siedlungstypen der französischen und italienischen Schweiz weisen deutlich geringere Geschlechterungleichheiten auf und eine wesentlich gleichstellungsfreundlicher eingestellte Stimmbevölkerung als die Pendlereinzugsgebiete der deutschschweizer Kernstädte. Ein höherer regionalwirtschaftlicher Entwicklungsstand schlägt sich somit nicht «automatisch» in geringeren strukturellen Geschlechterungleichheiten und in einer «fortschrittlicheren» Einstellung der Bevölkerung nieder.

Diese Besonderheiten einzelner Regionen ändern jedoch nichts an der Feststellung, dass insgesamt ein deutlicher Zusammenhang zwischen regionaler Geschlechterkultur und regionaler Geschlechterordnung besteht. Wichtig ist dabei zu erkennen, dass Geschlechterkultur und Geschlechterordnung einander gegenseitig beeinflussen und im Prozess des Arrangements miteinander in Beziehung gesetzt werden (siehe Punkt 2.1). Aus diesem Grunde ist es auch nicht möglich, eine zentrale Ursache für die unterschiedlichen Geschlechterverhältnisse in den einzelnen Regionen anzuführen. Das Geschlechterverhältnis in einer bestimmten Region ist ein ausgesprochen komplexes, multidimensionales soziales Phänomen, das durch vielfältige und rückgekoppelte kulturelle, ökonomische, politische und soziale Prozesse zustande kommt. Diese Prozesse in den einzelnen Regionen vertiefter zu analysieren, ist Aufgabe weiterführender Untersuchungen.

Aus dem vorliegenden Beitrag geht jedoch klar hervor, dass sich Geschlechterordnung und Geschlechterkultur in den verschiedenen Regionen der Schweiz zum Teil deutlich voneinander unterscheiden. Eine Ausweitung der vergleichenden Perspektive auf einen europäischen oder gar globalen Massstab würde noch wesentlich weitreichendere Unterschiede zu Tage bringen (siehe z. B. Duncan (1994) sowie Seager (1997)). Damit verliert eine bestimmte lokale/ regionale Geschlechterordnung bzw. eine spezifische, lokal/regional vorherrschende Geschlechterkultur jede Selbstverständlichkeit oder gar «Natürlichkeit». Das Geschlechterverhältnis in einer bestimmten Gesellschaft ist somit ein durch und durch soziales Phäno- men. Das noch relativ junge inter- und transdisziplinäre Forschungsgebiet der Gender Studies weist heute ein umfangreiches Spektrum theoretisch und empirisch spannender Fragestellungen auf, zu deren Beantwortung auch die Geographie ihren Beitrag leisten kann.

\section{Literatur}

Aregger, J. (1998): Presse, Geschlecht, Politik. Gleichstellungsdiskurs in der Schweizer Presse. $-=$ Berner Texte zur Medienwissenschaft 3, Bern: Institut für Medienwissenschaft.

Aufhauser, E. et al. (1999): FrauenArbeitMachtKörperRaum. Diskurse am Treffpunkt von Feminismus und Geographie. - In: BirKHAN, I. et al. (Hrsg.): Standpunkte feministischer Forschung und Lehre. $-=$ Materialien zur Förderung von Frauen in der Wissenschaft 9, Wien: 205-232.

BRÜCKNER, M. (1998):Wenn Forschende und Beforschte ein Geschlecht haben. Epistemologische, theoretische und methodologische Überlegungen. - In: Bulletin des Nationalen Forschungsprogrammes 40 «Gewalt im Alltag und organisierte Kriminalität» 2: 7-29.

BÜHLER, E. (2001): Frauen- und Gleichstellungsatlas Schweiz. $-=$ Gesellschaftsanalysen Schweiz 2, Zürich: Seismo.

Bundesamt für Statistik (1996): Auf dem Weg zur Gleichstellung? Frauen und Männer in der Schweiz. = Statistik der Schweiz Zweiter statistischer Bericht, Bern: Bundesamt für Statistik.

BUNDESAMT FÜR StatistiK (1999): Unbezahlt - aber trotzdem Arbeit. Zeitaufwand für Haus- und Familienarbeit, Ehrenamt, Freiwilligenarbeit und Nachbarschaftshilfe. $-=$ Sozialberichterstattung Schweiz, Neuchâtel: Bundesamt für Statistik.

Bundesamt für Statistik (2000): Maternité, mariage et divorce dans les années 90: quelles conséquences sur la vie professionnelle des femmes? - = SAKE-NEWS 4/2000, Bern: Bundesamt für Statistik.

Duncan, S. (1994): The diverse worlds of European patriarchy - guest editorial. - In: Environment and Planning A 26/8: 1174-1176.

DunCan, S. (1995): Theorising European Gender Systems. - In: Journal of European Social Policy 5/4: 263-284.

EIDGENÖSSISCHE KOMMISSION Für FRAUENFRAGEN (Hrsg.) (1998): Frauen Macht Geschichte. Frauenund gleichstellungspolitische Ereignisse in der Schweiz 1848-1998. - Bern: EDMZ.

EuropäISCHE Kommission (1997): Chancengleichheit von Frauen und Männern in der Europäischen Union - Jahresbericht 1996. - Luxemburg: Amt für amtliche Veröffentlichungen.

European Commission (1999): Equal opportunities for women and men in the European Union - Annual Report 1998. - Luxembourg: Office for Official Publications of the European Communities. 
Gilbert, A.-F. (1993): Feministische Geographien Ein Streifzug in die Zukunft. - In: BüHLER, E. et al. (Hrsg.): Ortssuche - zur Geographie der Geschlechterdifferenz. - = Schriftenreihe des Vereins Feministische Wissenschaft, Zürich, Dortmund: 79-108.

Harding, S. (1991): Feministische Wissenschaftstheorie. Zum Verhältnis von Wissenschaft und sozialem Geschlecht. - Hamburg: Argument.

Hardmeier, S. (1997): Frühe Frauenstimmrechtsbewegung in der Schweiz (1890-1930). Argumente, Strategien, Netzwerk und Gegenbewegungen. - Zürich: Chronos.

Hermann, M. (1998): Der Weltanschauungsraum - ein Instrument zur integrativen Erfassung mentaler, sozialer und regionaler Differenzierungen. - Zürich: Geographisches Institut, Universität Zürich.

JORIS, E. \& R. WECKER (1998): Mutterschaftsregelungen und Arbeit auf Abruf. Historische Beispiele und ihre aktuelle Bedeutung. - In: BüHLER, E. et al. (Hrsg.): Deregulierung und Chancengleichheit. Neue Herausforderung an Staat und Gesellschaft. - Zürich: 189-222. KLinger, C. (1994): Die politische Theoriediskussion der Gegenwart in einer feministischen Perspektive. In: KrIESI, H. (Hrsg.): Frauen und Politik. - = Schweizerisches Jahrbuch für politische Wissenschaft 34 , Bern: 33-50.

Kriesı, H. et al. (1996): Le clivage linguistique. Problèmes de compréhension entre les communautés linguistiques en Suisse. - = Statistique de la Suisse - culture, conditions de vie et sport, Bern: Bundesamt für Statistik.

Levy, R. et al. (1998): Alle gleich? Soziale Schichtung, Verhalten und Wahrnehmung. - Zürich: Seismo.

McDowell, L. (1999): Gender, Identity and Place. Understanding Feminist Geographies. - Cambridge: Polity Press.

Meuli, U. \& A. Ladner (2000): Frauen in den Gemeindeexekutiven. - = Kleine Zürcher Gemeindestudien, Zürich: Soziologisches Institut der Universität Zürich. Perrons, D. (1994): Measuring equal opportunities in European employment. - In: Environment and Planning A 26/8: 1195-1220.

Pfau-Effinger, B. (1996): Analyse internationaler Differenzen in der Erwerbsbeteiligung von Frauen. Theoretischer Rahmen und empirische Ergebnisse. - In: Kölner Zeitschrift für Soziologie und Sozialpsychologie 48/3: 462-492.

Pfau-Effinger, B. (1998): Gender Cultures and the Gender Arrangement - A Theoretical Framework for Cross-National Gender Research. - In: Innovation:The European Journal of Social Sciences 11/2: 147-166.

Pfau-EfFinger, B. (1999): Kultur, Wohlfahrtsstaat und Frauenarbeit im europäischen Vergleich. - Opladen: Leske und Budrich.

Schmid, H. et al. (1999): Monetäre Bewertung der unbezahlten Arbeit. Eine empirische Analyse für die Schweiz anhand der Schweizerischen Arbeits- kräfteerhebung.--= Statistik der Schweiz 16, Neuchâtel: Bundesamt für Statistik.

Schweizerische Zeitschrift für Geschichte (1996): Thema Geschlecht und Staat. - = Schweizerische Zeitschrift für Geschichte 46/3.

Schweizerischer Bundesrat (1999): Stellungnahme des Bundesrates zur Abstimmung über die Mutterschaftsversicherung am 13. Juni 1999. - Bern.

Seager, J. (1997): The State of the Women in the World Atlas. Women's Status around the Globe: Work, Health, Education and Personal Freedom. - = Penguin Reference, London: Penguin Books.

Sertz, W. (1994): Die Frauen bei den Nationalratswahlen 1971-1991 aus statistischer Sicht. - In: KrIEsI, H. (Hrsg.): Frauen und Politik. - = Schweizerisches Jahrbuch für Politische Wissenschaft 34, Bern: 225-249.

Senti, M. (1994): Geschlecht als politischer Konflikt - Erfolgsbedingungen einer gleichstellungspolitischen Interessensdurchsetzung. Eine empirische Untersuchung am Beispiel der Schweiz. - = Berner Studien zur Politikwissenschaft Band 2, Bern: Paul Haupt.

Studer, B. (1996): L'Etat c'est l'homme. Politique, citoyenneté et genre dans le débat autour du suffrage féminin après 1945. - In: Schweizerische Zeitschrift für Geschichte 46/3: 356-382.

Studer, B. et al.(Hrsg.) (1998):Frauen und Staat. Berichte des Schweizerischen Historikertages vom Oktober 1996 in Bern. - = ITINERA 20, Basel: Schwabe \& Co. AG. UNDP (jährlich): Human Development Report. Oxford: United Nations Organisation.

Voegell, Y. (1997): Zwischen Hausrat und Rathaus. Auseinandersetzungen um die politische Gleichberechtigung der Frauen in der Schweiz 1945-1971. Zürich: Chronos.

WALBY,S. (1993): Neue theoretische Ansätze zur Untersuchung des Geschlechterverhältnisses. - In: BüHLER, E. et al. (Hrsg.): Ortssuche - Zur Geographie der Geschlechterdifferenz. - = Schriftenreihe des Vereins Feministische Wissenschaft, Zürich/Dortmund: 49-78. WALBY,S. (1994): Methodological and theoretical issues in the comparative analysis of gender relations in Western Europe. - In: Environment and Planning A 26/9: 1339-1354.

WeEdon, C. (1991): Wissen und Erfahrung: feministische Praxis und poststrukturalistische Theorie. Zumikon/Dortmund: eFeF-Verlag.

Women and Geography Study Group (1997): Feminist Geographies: Explorations in Diversity and Difference. - London: Longman.

\section{Zusammenfassung: Zum Verhältnis von kulturellen Werten und gesellschaftlichen Strukturen in der Schweiz - das Beispiel regionaler Gemeinsamkeiten und Differenzen der Geschlechterungleichheit} Das Geschlechterverhältnis an einem bestimmten Ort kann gemäss neueren Theorien der Geschlechterfor- 
schung (Gender Studies) als Resultat eines Aushandlungs- und Kompromissbildungsprozesses zwischen gesellschaftlichen Akteurinnen und Akteuren gedeutet werden. In diesem Arrangement werden die Werte und Leitbilder der Menschen über eine angemessene, «richtige» Rollenteilung zwischen Frau und Mann (Geschlechterkultur) mit den wirtschaftlichen, politischen und anderen institutionellen Strukturen des Geschlechterverhältnisses (Geschlechterordnung) miteinander in Beziehung gesetzt und in ein mehr oder weniger hohes Mass an Übereinstimmung gebracht. Eine regional differenzierte Auswertung sekundärstatistischer Daten (eidgenössische Volksund Betriebszählungen sowie Volksabstimmungen) belegt, dass in der Schweiz sowohl in Bezug auf die regional vorherrschende Geschlechterkultur als auch in Bezug auf die Geschlechterordnung teilweise beträchtliche regionale Differenzen vorhanden sind. Dabei besteht ein deutlich erkennbarer Zusammenhang zwischen regionaler Geschlechterkultur und regionaler Geschlechterordnung. Dort, wo die Ergebnisse der Volksabstimmungen insgesamt eine gleichstellungsfreundlichere Einstellung der Stimmbevölkerung zum Ausdruck bringen, ist auch die Geschlechterungleichheit insgesamt relativ geringer als in Regionen mit einer weniger gleichstellungsfreundlich eingestellten Stimmbevölkerung. Die Ergebnisse dieser vom Schweizerischen Nationalfonds finanzierten Studie stützen die Erkenntnis, dass das Verhältnis der Geschlechter ein soziales und kulturell variables und kein «natürliches» oder selbstverständliches Phänomen darstellt.

\section{Summary: On the Relationship between Cultural Values and Social Structures in Switzerland - the example of regional similarities and differences in gender inequality}

According to recent theories, gender relations in a specific place can be understood as a result of negotiation processes and struggles between social actors. These arrangements reflect a more or less satisfying compromise between the dominant norms and values of the social actors about the desirable forms of gender relations (gender culture) and economic, political and other institutional structures with respect to gender relations (gender order). A regionally differentiated analysis of secondary statistical data (federal population and occupation census, results of federal votes) shows that there are remarkable regional differences in Switzerland concerning dominant regional gender cultures and regional gender orders. At the same time a significant correlation between regional gender culture and regional gender order can be observed. In regions, where the results of the federal votes reveal a more favourable attitude of the voters for equal opportunity issues, gender inequality in the different social institutions is smaller than in regions with a less favourable attitude of the voters towards equal opportunity issues. These results of a study financed by the Swiss National Science Foundation support the notion that gender relationship is a social and cultural variable and not a «natural» or self-evident phenomenon.

\section{Résumé: Relation entre les valeurs culturelles et les structures sociales en Suisse - l'exemple des simi- litudes et des différences régionales ayant trait à l'inégalité entre les sexes}

Selon les dernières théories des études sur le genre (Gender Studies), le rapport entre les sexes en un lieu donné peut s'interpréter comme le résultat d'un processus de négociation et de compromis entre des actrices et des acteurs sociaux. Dans cet «Arrangement», les valeurs et les modèles de l'être humain relatifs à une répartition des rôles appropriée et «correcte» entre la femme et l'homme (Gender Culture) sont mis en relation avec les structures institutionnelles économiques, politiques ou autres du rapport entre les sexes (Gender order) et plus ou moins accordés. Une analyse par régions de données statistiques d'ordre secondaire (recensements fédéraux de la population et des entreprises, votations populaires) révèle que la culture relative au genre qui prédomine au niveau régional varie souvent considérablement selon des régions du pays, de même que l'ordre relatif au genre. On relève un lien très net entre culture régionale et ordre régional. En général, l'inégalité entre les sexes est relativement moins importante dans les régions où les résultats des votations populaires reflètent une attitude plus favorable du souverain par rapport à l'égalité entre les sexes que dans les régions comptant un électorat moins favorable à cette égalité. Les conclusions de cette étude financée par le Fonds national suisse étayent ce qui a déjà été dit sur le sujet: le rapport entre les sexes constitue une variable sociale et culturelle et non un phénomène «naturel» allant de soi.

Dr. Elisabeth Bühler, Abteilung Wirtschaftsgeographie, Geographisches Institut, Universität Zürich, Winterthurerstr. 190, CH-8057 Zürich.

e-mail: buehler@geo.unizh.ch

Manuskripteingang/received/rentrée du mansucrit: 21.12.2000

Annahme zum Druck/Accepted for publication/ acceptation à l'impression: 21.6.2001 